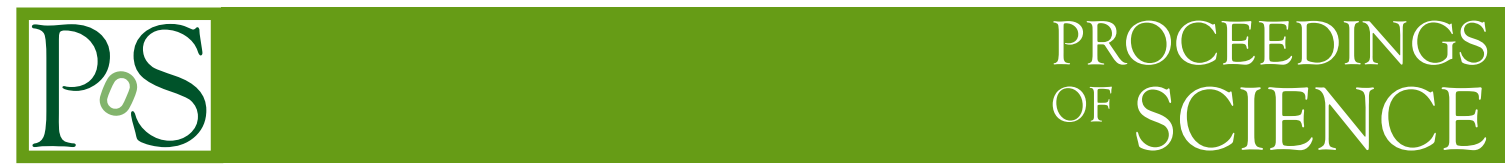

\title{
Quantum field theory on a causal set
}

\author{
Roman Sverdlov * \\ Department of Mathematics, University of New Mexico \\ E-mail: rsverdlov@unm.edu
}

Causal set theory, originally introduced by Rafael Sorkin, is a model of spacetime as a partially ordered set: an element of a set corresponds to a point in spacetime, while partial ordering corresponds to lightcone causal relation. There is no coordinate system: all of the geometry is to be deduced from partial ordering alone. Consequently, one has to rewrite Lagrangians in quantum field theory in such a way that would avoid derivative signs or anything else with Lorentz index. In my talk I will discuss some of the ways of doing so (both the ones introduced by myself and by others).

The 36th Annual International Symposium on Lattice Field Theory - LATTICE2018

22-28 July, 2018

Michigan State University, East Lansing, Michigan, USA.

${ }^{*}$ Speaker. 


\section{Introduction}

A causal set is a model of discrete spacetime as partially ordered set: $a \prec b$ if and only if one can go from $a$ to $b$ without going faster than the speed of light. It has a topology given by Alexandrov sets,

$$
\alpha(p, q)=\{r \mid p \prec r \prec q\}
$$

and we say $p \prec^{*} q$ if $\alpha(p, q)$ is empty (in other words, $\prec^{*}$ represents a direct neighbor relation). Geometrically, Alexandrov sets are diamonds (see pic 1 on p.1 of [8]) and discreteness is postulated through the assertion that they have finitely many elements (see pic 2 on p.1 of [8]). A distance function is defined by

$$
\tau(a, b)=\xi \max \left\{n \mid \exists c_{1}, \cdots, c_{n-1}\left(a \prec c_{1} \prec \cdots \prec c_{n-1} \prec b\right)\right\}
$$

where $\xi$ is a Planck length, and we are taking maximum instead of the minimum in order to reflect the fact that, in Lorentzian case, a timelike geodesic is the longest curve rather than shortest curve (see pic 3 on p.1 of [8]). The goal of causal set theory is to re-express key concepts that are used in physics (fields, Lagrangians, etc) in terms of the above entities in coordinate-independent way, so that they continue to be well defined when manifold structure breaks down. In this talk I will compare my own approaches to causal sets ( $\operatorname{Sec} 4, \operatorname{Sec} 5$ and $\operatorname{Sec} 6$ ) to the ones of other people (Sec 1, 2 and $\operatorname{Sec} 3)$.

\section{Conventional version of causal set Lagrangian}

Before I talk about my own work (which takes totally different approach) let me first summarize the approach used by others. The most conventional approach to scalar fields on causal sets, developed by Sorkin, Dawker and others, is based on D'Ambertians (for most recent review, see [1]) By using integration by parts, they choose to use $-\phi \Delta \phi$ instead of $+\partial^{\mu} \phi \partial_{\mu} \phi$; then they model $\Delta \phi$ in terms of the sums. In 2 dimensional situation, the sum takes the form

$$
(\Delta \phi)(p)=\sum_{\{(r, s) \mid \alpha(r, p)=\{s\}\}}(\phi(p)+\phi(r)-2 \phi(s))
$$

while in more general dimension it takes the form of

$$
\Delta \phi=\sum_{r_{n(d)} \prec^{*} r_{n(d)-1} \prec^{*} \ldots \prec^{*} r_{1} \prec^{*} p}\left(c_{0}(d) \phi(p)+c_{1}(d) \phi\left(r_{1}\right)+\cdots+c_{n(d)}(d) \phi\left(r_{n(d)}\right)\right)
$$

where $\mathrm{n}(\mathrm{d})$ is some integer associated with $d$ and $c_{0}(d)+\cdots+c_{n(d)}(d)=0$. Even in $d=2$ case of Eq 2.1 (let alone Eq 2.2) we get infinitely many terms due to the fact that most of the "direct neighbors" of any given point are arbitrary close to its light cone, and arbitrary far coordinate-wise (see p.3 of [8]). However, if we assume spacetime is finite and, at the same time add Lagrangian densities at different nearby points, then the contributions to the Lagrangian coming from coordinate neighborhood would add up, while the contributions that are coordinate-wise far away would cancel each other out (see p.3 of [8]), which would result in better and better approximation of the continuum Lagrangian. Be it as it may, the fact that we would have $\infty-\infty$ in case of infinite spacetime makes me uncomfortable, and my own work is focused on alternative models that avoid this feature. 


\section{Steven Johnston's propagator}

Stephen Johnston proposed a different approach (see [2]), where instead of focusing on Lagrangian density he goes straight to the propagator. Even though he is doing quantum field theory as opposed to quantum mechanics, he is performing path integral over trajectories of point particles as opposed to the trajectories of the field. The particle undergoes a series of "hops" and "stops" (see pic 1 on p.4 of [8]) each of which is assigned complex valued probability. In case of twopoint function, $D(a, b)$, the nonlocality is avoided naturally, since the only spacetime region that is relevent is $\alpha(a, b)$, whose volume is finite. He haven't discussed how to treat the $\phi^{4}$ case, but I can think of some simple additions to his theory that would take care of $\phi^{4}$. For example, in case of $\phi^{4}$ -coupling between the propagators $D(a, e), D(b, e), D(c, e)$ and $D(d, e)$, I propose to impose upper bound on the distance between them (see pic 2 on p.4 of [8]). One option is to assume that they are causally related - which, without loss of generality, can be $a \prec b \prec c \prec d \prec e$ and then impose upper bound $\tau(a, e)<\Lambda$, for some large $\Lambda$. Other kinds of 4- verteces can be treated similarly.

\section{Electromagnetic Lagrangian}

Let us now turn to my own work. I define electromagnetic field on a causal set to be a two-point function

$$
a(r, s)=\int_{\gamma(r, s)} A_{\mu} d x^{\mu}
$$

where $\gamma(r, s)$ is a geodesic connecting $r$ and $s$. We assume that $A_{\mu}$ is approximately linear over the Alexandrov set $\alpha(p, q)$ and, based on this assumption, we would like to find an approximation to the Lagrangian density in the interior of that set. We then define Lagrangian generators as

$$
\begin{aligned}
& \mathscr{K}_{1}=\int_{\alpha(p, q)} d^{d} r d^{d} s(a(p, r)+a(r, q)+a(q, s)+a(s, p))^{2} \\
& \mathscr{K}_{2}=\int_{\alpha(p, q)} d^{d} r d^{d} s(a(p, r)+a(r, s)+a(s, q)+a(q, p))^{2}
\end{aligned}
$$

which correspond to the contours drawn on p.11 of [8]. If we select a reference frame in which $t$-axis coincides with the line passing from $p$ to $q$, one can show that

$$
\begin{gathered}
\mathscr{K}_{1}=k(d) \tau^{2 d+2}(p, q)|\vec{E}|^{2} \\
\mathscr{K}_{2}=\tau^{2 d+2}(p, q)\left(A_{d}|\vec{E}|^{2}+B_{d}|\vec{B}|^{2}\right)
\end{gathered}
$$

and, therefore, one can find $\eta_{\text {scal }}(d)$ and $C(d)$ such that

$$
\mathscr{L}=|\vec{E}|^{2}-|\vec{B}|^{2}=\eta_{\text {scal }}(d)\left(\mathscr{K}_{1}(\phi ; p, q, r)-C_{\text {scal }}(d) \mathscr{K}_{2}(\phi ; p, q)\right)
$$

In [4] the value of $C(d)$ was computed, which turns out to be very complicated.

There is an alternative approach, presented in [5], that is meant to remove the explicit $d$ dependence, and it leads to a much simpler formula. Lets assume that we don't know what $d$ is. In this case we can find $C(d)$ (and, if we like, invert the formulas in [4] to find $d$ ) by the use of "test functions". Suppose we have a test function $b$ that is assumed to be linear, and suppose we 
have two Alexandrov sets, $\alpha\left(p_{1}, q_{1}\right)$ and $\alpha\left(p_{2}, q_{2}\right)$, such that $\tau\left(p_{1}, q_{1}\right)=\tau\left(p_{2}, q_{2}\right)$ with common midpoint (see the middle of the drawing on p.9 on [8]). Since those Alexandrov sets are simply Lorentz boosts of one another, we would expect the Lagrangian density estimates corresponding to those sets to be the same. In other words, we would expect the equation

$$
\mathscr{K}_{1}\left(b ; p_{1}, q_{1}\right)-C(d) \mathscr{K}_{2}\left(b ; p_{1}, q_{1}\right) \approx \mathscr{K}_{1}\left(b ; p_{2}, q_{2}\right)-C(d) \mathscr{K}_{2}\left(b ; p_{2}, q_{2}\right)
$$

to hold. This implies

$$
C(d) \approx \frac{\mathscr{K}_{1}\left(b ; p_{1}, q_{1}\right)-\mathscr{K}_{1}\left(b ; p_{2}, q_{2}\right)}{\mathscr{K}_{2}\left(b ; p_{1}, q_{1}\right)-\mathscr{K}_{2}\left(b ; p_{2}, q_{2}\right)}
$$

where, notably, the right hand side lacks explicit $d$-dependence, which means that $C(d)$ gets replaced with $C(b)$. Since we would like Lagrangian to be quadratic function, we make a distinction between test function $b$, which is to be fixed, and gauge field $a$, which is to vary. The Lagrangian density of $a$ over an Alexandrov set $\alpha\left(p_{0}, q_{0}\right)$ (depicted at the top of the drawing on p.9 on [8]) is given by

$$
\mathscr{L}=\eta\left(\mathscr{K}_{1}\left(a ; p_{0}, q_{0}, r\right)-C(b) \mathscr{K}_{2}\left(a ; p_{0}, q_{0}\right)\right)
$$

where $C(b)$ is given by the right hand side of Eq 4.8. Now, if we use just one test function, it would imply a preferred frame (for example, a direction of vector potential associated with that test function, if it happens to be timelike, among various other things one can derive). So we replace it with a weighted average taken over several test functions:

$$
\mathscr{L}=\sum_{b} N(b)\left(\mathscr{K}_{1}\left(a ; p_{0}, q_{0}, r\right)-C(b) \mathscr{K}_{2}\left(a ; p_{0}, q_{0}\right)\right)
$$

If we define $M(b)$ to be

$$
M(b)=\frac{N(b)}{\mathscr{K}_{2}\left(b ; p_{1}, q_{1}\right)-\mathscr{K}_{2}\left(b ; p_{2}, q_{2}\right)}
$$

and substitute Eq 4.8, we obtain

$$
\begin{aligned}
\mathscr{L}\left(a ; p_{0}, q_{0}\right)= & \sum_{b}\left(M ( b ) \left(\mathscr { K } _ { 1 } ( a ; p _ { 0 } , q _ { 0 } ) \left(\mathscr{K}_{2}\left(b ; p_{1}, q_{1}\right)\right.\right.\right. \\
& \left.\left.\left.-\mathscr{K}_{2}\left(b ; p_{2}, q_{2}\right)\right)-\mathscr{K}_{2}\left(a ; p_{0}, q_{0}\right)\left(\mathscr{K}_{1}\left(b ; p_{1}, q_{1}\right)-\mathscr{K}_{1}\left(b ; p_{2}, q_{2}\right)\right)\right)\right)
\end{aligned}
$$

Now, in order for the sum over $b$ to be finite rather than infinite, we say that $b$ is being produced by imaginary "source" $\left(p_{3}, q_{3}\right)$ (depicted at the bottom of the drawing on p.9 of [8]) and then we replace $M(b)$ with $M\left(p_{0}, q_{0}, p_{1}, q_{1}, p_{2}, q_{2}, p_{3}, q_{3}\right)$; thus,

$$
\begin{aligned}
\mathscr{L}\left(\phi ; p_{0}, q_{0}\right)= & \sum_{p_{3} q_{3}}\left(M\left(p_{0}, q_{0}, p_{1}, q_{1}, p_{2}, q_{2}, p_{3}, q_{3}\right) \times\right. \\
& \times\left(\mathscr{K}_{1}\left(\phi ; p_{0}, q_{0}\right)\left(\mathscr{K}_{2}\left(b_{p_{3} q_{3}} ; p_{1}, q_{1}\right)-\mathscr{K}_{2}\left(b_{p_{3} q_{3}} ; p_{2}, q_{2}\right)\right)\right. \\
& \left.\left.-\mathscr{K}_{2}\left(\phi ; p_{0}, q_{0}\right)\left(\mathscr{K}_{1}\left(b_{p_{3} q_{3}} ; p_{1}, q_{1}\right)-\mathscr{K}_{1}\left(b_{p_{3} q_{3}} ; p_{2}, q_{2}\right)\right)\right)\right)
\end{aligned}
$$

where $b_{p_{3} q_{3}}$ is the test function sourced at $\left(p_{3}, q_{3}\right)$. The above will have finitely many terms since we can simply put an upper bound on the distances from $p_{3}$ and $q_{3}$ to other points. The choice 
of that upper bound, as well as the specific correspondence between the source $\left(p_{3}, q_{3}\right)$ and the resulting $b_{p_{3} q_{3}}$ is not very important, as long as those things are well defined, smooth, and the unwanted effects of curvature among other things are avoided. The most convenient way of defining $b_{p_{3} q_{3}}(p, q)$ is to come up with some arbitrary function of distances from points $p$ and $q$ to points $p_{3}$ and $q_{3}$; an example of such function is presented in [4].

\section{Locality and Lorentz invariance issues}

After reading the last section one can ask the following question: why did we need to adjust coefficients and why didn't Lorentz covariance arize naturally out of the fact that partial ordering is manifestly invariant? The answer to this question is that the choice of Alexandrov set broke the invariance. On the other hand, in Section 2 the Alexandrov set wasn't used and, therefore, Lorentz invariance was preserved. However, in that situation a different problem arizes: if the universe is infinite, the cancellation we hope to get from Eq 2.1 and Eq 2.2 is $\infty-\infty$ which isn't mathematically well defined. If, on the other hand, the universe is finite, then its shape would select preferred frame. Thus, no matter what we do, we end up with finite region that allows us to avoid infinity at the price of emergence of preferred frame; in case of Sec 2, that region is the whole universe that is declared to be finite, in case of Sec 4 it is Alexandrov set. But the end result of violating invariance is the same. In [3] it is also argued that similar issue arises as a result of presence of discretization itself. If neighboring of a point is local - as it is in Eucledian case - then the direction to nearest neighbor will lead to preferred frame (see pic 2 on p.5 of [8]). If the neighborhood is nonlocal, as is the case in Minkowskian space, then we get infinitely many neighbors along the vicinity of the light cone, none of them will be nearest (see p.3 of [8]). Thus, once again, there is a trade-off between avoiding nonlocality and avoiding preferred frame: we can avoid one or the other, but not both.

In this paper I propose to avoid this trade-off by thinking in terms of tangent bundle instead of a manifold. Thus, a generic field $\mathscr{F}$ is not $\mathscr{F}(x)$ but rather $\mathscr{F}(x, v)$, and the Lagrangian density is not $\mathscr{L}(\mathscr{F}, x)$ but rather $\mathscr{L}(\mathscr{F}, x, v)$, where $x$ is a point on a manifold, and $v$ is a timelike tangent vector at $x$. In case of $\mathscr{F}(x, v)$ we do that by attaching $\mathscr{F}$ to an edge rather than a point, and thinking of $v$ as a direction of that edge; in case of $\mathscr{L}(\mathscr{F}, x, v)$ we think of $v$ as a direction of the line connecting the endpoints of Alexandrov set (assuming the spacetime is locally flat). In case of gauge field, we have to attach it to edges anyway. In case of scalar field, however, it would have been more natural to attach it to a point and have $\phi(r)$. But, for the purposes of the above, we attach it to the edge by defining

$$
\phi(r, s)=\frac{1}{\tau(r, s)} \int_{\gamma(r, s)} \phi(x)|d x|
$$

where $\gamma(r, s)$ is a geodesic connecting $r$ and $s$. As far as discussion in [3], we can argue that the "preferred direction" in $(x, v)$-case that would arize as a cost of locality corresponds to acceleration. Preferred acceleration can be explained away through gravity, which can't be said about preferred velocity.

There is another alternative: instead of using $\phi(r, s)$ we can use $\phi(r)$ but interpret $r$ as $(x, v)$ rather than $x$. This can be accomplished in several ways. One way is to first do Poisson scattering over a manifold and then, at each of the produced points, do the Poisson scattering on corresponding 
tangent plane, and the other way is to simply do Poisson scattering over the tangent bundle instead of the manifold and then a single Poisson scattering can suffice (these two ways are, respectively, portrayed on left side and right side of drawing on (see the left side of drawing on p.13 of [8]). This also involves introducing new type of causal relation: $(x, u) \prec(y, v)$ if one can start at $x$, with velocity $u$, and reach $y$, with velocity $v$, without Lorentzian acceleration exceeding $a_{\max }$ along the way. This causal relation is depicted in the drawing on top right corner of p.12 on [8], where red points are the ones causally related to the origin and green points are the ones causally unrelated. This causal relation is contrasted with more common, $x$-only one, depicted at the top left corner.

\section{Building wavelets}

Let us now describe an alternative way of introducing scalar field. In previous section we were assuming that $\phi(x, v) \approx \phi(x)$, unless $v$ is close to the speed of light. Let us switch to a different assumption; namely, $\phi(x, v) \approx \tilde{\phi}(\mu v)$ where $\tilde{\phi}$ is a Fourier transform of $\phi$, and $\mu$ is some scalar. In order to accomplish this, we would ideally want the derivative of $\phi$ to be zero in $x$-directions orthogonal to $v$. But, in light of spacetime curvature, this can't be consistently implemented. So, instead, we postulate a constraint

$$
\Delta_{\perp} \phi=-\varepsilon(R) \phi
$$

where $\Delta_{\perp}$ is a Laplacian with respect to an $x$-based hyperplane that is perpendicular to the direction of $v, R$ is Ricci curvature and $\varepsilon$ is some function satisfying

$$
\varepsilon(0)=0
$$

In light of discreteness, we define $\partial_{\|}$and $\Delta_{\perp}$ in an integral form (by means of heat kernels) and then replace integrals with the sums:

$$
\begin{gathered}
\partial_{\|} \phi=\frac{\sum_{\left(p^{\prime}, q^{\prime}\right) \in \|_{\chi}(p, q)}\left(\phi\left(p^{\prime}, q^{\prime}\right)-\phi(p, q)\right)}{\sum_{\left(p^{\prime \prime}, q^{\prime \prime}\right) \in \|_{\chi}(p, q)} 1} \\
\Delta_{\perp} \phi=\frac{2 \alpha \sum_{\left(p^{\prime}, q^{\prime}\right) \in \perp_{\delta}(p, q)}\left(\phi\left(p^{\prime}, q^{\prime}\right)-\phi(p, q)\right) e^{-\alpha d^{2}\left((p, q),\left(p^{\prime}, q^{\prime}\right)\right) / 2}}{\sum_{\left(p^{\prime \prime}, q^{\prime \prime}\right) \in \perp_{\delta}(p, q)} e^{-\alpha d^{2}\left((p, q),\left(p^{\prime \prime}, q^{\prime \prime}\right)\right) / 2}}
\end{gathered}
$$

where $\|_{\chi}(p, q)$ is a set of edges that can be produced by displacing the edge $(p, q)$ by a distance $\chi$ in a direction parallel to itself (and that set can, potentially, have more than one element due to discreteness) while $\perp_{\delta}(p, q)$ is a set of edges that can be produced by displacement of an edge $(p, q)$ by a distance $\delta$ in a direction perpendicular to itself.

This, however, leads to the question: how do we define parallel transport? Indeed, one can see that if the edges are drawn between the direct neighbors, we wouldn't statistically expect to find any parallel ones. In order to fix the situation we replace "short edges" with "long edges", where by "long" we mean much longer than the scale of the laboratory (see drawing on p.15 on [8] and also the top right of p.16 of [8]). This does not compromise the locality in $x$ because we assume that $\phi$ is "concentrated" at the "future-most end" of each edge. In other words, if $\gamma$ is a geodesic connecting $p$ and $q$ then the pair $(p, q)$ is identified with a pair $(q, v)$ where $v$ is a tangent vector to $\gamma$ at $q$ :

$$
\phi(p, q)=\phi(q, v) \text { where } p=\gamma(0), q=\gamma(1), v=\gamma^{\prime}(1)
$$


Despite the fact that the distance between $p$ and $q$ is larger than the size of the laboratory, the right hand side is local since $p$ doesn't occur on the right hand side, and $v$ is a tangent vector at $q$. Finally, the notion of "size of the laboratory" should be replaced with something more physical. From the above discussion, one can see that "laboratory" is a region where we insist that plane wave is preserved up to some approximation. In other words, the effects of gravitational field as well as $\phi^{4}$-coupling aren't felt on that scale. That would be true if the size of the laboratory satisfies

$$
L_{L a b} \ll \min \left(\frac{1}{R}, \frac{m^{2}}{\lambda \phi_{\min }^{2}}\right)
$$

where $\lambda$ is a $\phi^{4}$-coupling constant and $\phi_{\min }$ is the smallest detectable value of $\phi$. We then guarantee $\tau_{1} \gg L_{L a b}$ by postulating a "stronger" condition,

$$
\tau_{1} \gg \min \left(\frac{1}{R}, \frac{m^{2}}{\lambda \phi_{\min }^{2}}\right)
$$

\section{Conclusion}

In this paper we have outlined the work done by others (Sec 1, 2 and Sec 3) as well as the work done by the author of this paper (Sec 4, Sec 5 and $\operatorname{Sec} 6$ ) The key difference between my own work and the work done by others is that, in my own work, I have attempted to shift emphasis from the manifold itself to the tangent bundle in order to address locality, whereas other people's work was done within manifold context. However I believe that the work by Johnston (sec 3) can also be made local (without resorting to tangent bundle) by means of a modification that I proposed in that section.

\section{Acknowledgments}

The research of Roman Sverdlov was supported in part by NSF grant DMS-1554456 and DMS 9215024.

\section{References}

[1] Fay Dowker, Lisa Glaser "Causal set d'Alembertians for various dimensions" arXiv:grqc/0703099

[2] Steven Johnston "Particle propagators on discrete spacetime" arXiv:0806.3083

[3] L. Bombelli, J. Henson and R. Sorkin, "Discreteness without Symmetry Breaking: a Theorem," arXiv:gr-gc/060500v1

[4] Roman Sverdlov "Electromagnetic Lagrangian on a causal set that resides on edges rather than points" arXiv:1805.08064

[5] Roman Sverdlov "The use of test functions to help define quadratic Lagrangian on a causal set" arXiv: 1807.07403

[6] Roman Sverdlov "Causal set as a discretized phase spacetime" arXiv:0910.2498

[7] Roman Sverdlov "Restoring locality of scalar fields on a causal set by avoiding the use of d'Alembertians" arXiv:1805.11420

[8] https://indico.fnal.gov/event/15949/session/16/contribution/14/material/slides/0.pdf 